\title{
The advantages of carbon nanoparticles in level VII lymph node dissection in patients with papillary thyroid cancer
}

\author{
Yijun Chen, Guolie Zhang, Yuanmei Lin, Guoliang Zhang, Jian Gao \\ First Department of Thyroid Surgery, the Affiliated Hospital of Putian University, Putian, China \\ Contributions: (I) Conception and design: Y Chen, G Zhang, Y Lin, J Gao; (II) Administrative support: Y Lin; (III) Provision of study materials or \\ patients: Y Chen, G Zhang; (IV) Collection and assembly of data: J Gao; (V) Data analysis and interpretation: Y Chen, G Zhang; (VI) Manuscript \\ writing: All authors; (VII) Final approval of manuscript: All authors. \\ Correspondence to: Yijun Chen, MD. First Department of Thyroid Surgery, the Affiliated Hospital of Putian University, Putian 351100 , China. \\ Email: chenyijun1987@126.com.
}

\begin{abstract}
Background: The characteristics of level VII lymph nodes were explored and the advantages of using carbon nanoparticles (CNs) in papillary thyroid cancer (PTC) surgeries were examined. This was a retrospective study investigating the clinical data of patients with PTC who underwent surgical treatment.

Methods: From September 2019 to December 2020, a total of 191 consecutive patients with PTC were retrospectively analyzed. The patients were randomly divided into the CNs group and the control group. CNs were used in the CNs group during the operation. There were 103 cases in the CNs group, including 25 males and 78 females, and 88 cases in the control group, including 21 males and 67 females. Parameters, including the characteristics of dissected lymph nodes in level VII, metastatic rate, postoperative complications, and other inpatient-related indicators, were compared between the two groups.

Results: The number of lymph nodes dissected in level VII in the CNs group was significantly greater than that in the control group, and the metastatic rate of black-stained lymph nodes was significantly higher than that of non-black-stained lymph nodes $(\mathrm{P}<0.05)$. The postoperative hypoparathyroidism rate in the CNs group was significantly lower than that in the control group $(\mathrm{P}<0.05)$. There were no differences in the operation duration, intraoperative blood loss, postoperative drainage duration, or hospitalization days between the two groups $(\mathrm{P}>0.05)$.

Conclusions: The application of CNs for patients with PTC significantly increased the number of lymph nodes dissected in level VII and helped to protect the parathyroid glands (PGs). Moreover, the use of CNs did not increase the rate of postoperative complications. Thus, CNs may be a simple and safe approach when performing surgery in PTC patients.
\end{abstract}

Keywords: Carbon nanoparticles (CNs); level VII; papillary thyroid cancer (PTC); central lymph node dissection (CLND); hypoparathyroidism

Submitted Apr 07, 2021. Accepted for publication Jun 15, 2021.

doi: $10.21037 / g s-21-281$

View this article at: https://dx.doi.org/10.21037/gs-21-281

\section{Introduction}

The incidence of thyroid carcinoma (TC) has increased in recent years. It is the 11 th most common cancer globally and ranks 7 th among all malignant tumors in China (1). The most common pathological type of thyroid cancer is papillary thyroid cancer (PTC), making up about $85-90 \%$ of all TCs. Surgery is the most effective treatment for patients with PTC, and the postoperative overall survival can exceed $90 \%$ at 10 years $(2,3)$. Despite the good overall survival rates, cervical lymph node metastasis (CLNM) is a major risk factor for recurrence and distant metastasis of PTC and can severely affect patient prognosis $(4,5)$. Indeed, CLNM, especially in the central region, can reach a 


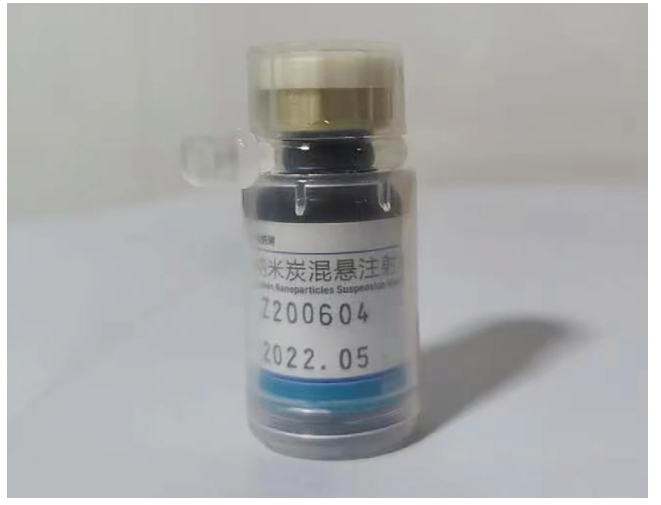

Figure 1 The image of Carbon Nanoparticles Suspension $(1 \mathrm{~mL}$ : 50 mg; Lai Mei Pharmaceutical Co, Chongqing, China).

metastasis rate as high as $20-90 \%(2,6,7)$.

In the past, most surgeons performed central lymph node dissection (CLND) exclusively in level VI. However, in recent years, it has been advocated that the anterior superior mediastinal lymph node (level VII) be classified as the central area of lymph node dissection (8-11). In fact, the $8^{\text {th }}$ American Joint Committee on Cancer (AJCC) classified this lymph node as a central region lymph node (12). Furthermore, the Chinese Thyroid Association (CTA) guidelines also suggest that PTC patients should receive routine CLND to reduce the risk of long-term recurrence. Hence, in our department, prophylactic or therapeutic CLND, including level VI and level VII, is routinely performed in all PTC patients.

To achieve a more radical effect, our department uses carbon nanoparticles (CNs) (Figure 1). CNs are kind of lymphatic tracer with a particle diameter of $150 \mathrm{~nm}$. They can enter the lymphatic system and trace the lymph nodes during CLND $(13,14)$. In PTC surgery, injection of CNs into tissues near the tumor can cause the central lymph node drainage to appear black. Thus, this technique can assist surgeons in determining the extent of CLND and prevent suspicious lymph nodes from being missed. Although there are some similar reports about the advantages of CNs used in PTC patients, there has been no related research on the use of CNs in level VII dissections. This study analyzed the effects of CNs used in level VII lymph nodes dissection with PTC patients. We present the following article in accordance with the STROBE reporting checklist (available at https://dx.doi. org/10.21037/gs-21-281).

\section{Methods}

\section{Patients}

This study investigated the clinical data of patients with PTC who underwent hemithyroidectomy/total thyroidectomy plus CLND in the Department of Thyroid Surgery of Putian University Affiliated Hospital between September 2019 and December 2020. All specimens were confirmed to be PTC by postoperative histopathology. All patients were informed of the methods and risks of the operation and signed informed consents. The patients were randomly divided into two groups. In the $\mathrm{CNs}$ group, $\mathrm{CNs}$ suspension (1 mL: $50 \mathrm{mg}$; Lai Mei Pharmaceutical Co, Chongqing, China) were partly injected into the thyroid gland of 103 patients. The remaining 88 patients were designated as the control group. All patients underwent surgery for the first time and were not complicated with malignant tumors of other organs. All patients were examined by thyroid ultrasound, cervical computed tomography (CT), and electronic laryngoscopy. Serum calcium and parathyroid hormone (PTH) levels, as well as thyroid function were assessed before surgery and were followed up for 6 months. Permanent hypoparathyroidism and recurrent laryngeal nerve (RLN) injury were defined if PTH levels and vocal cord movement did not return to normal after 6 months. All procedures performed in this study involving human participants were in accordance with the Declaration of Helsinki (as revised in 2013). This research was approved by the medical ethics committee of the Affiliated Hospital of Putian University.

\section{Definition of level VI and VII lymph nodes}

In this study, according to the NCCN guidelines, the scope of CLND included levels VI and VII. The scope of the dissection is detailed as follows: the lateral boundary consisted of the inner edge of the carotid sheath; the upper boundary consisted of the level of the hyoid bone; the lower boundary was defined by the innominate artery; and the posterior boundary was defined by the dorsal prevertebral fascia. The extent of lymph nodes in level VI included the anterior laryngeal, pretracheal, and paratracheal lymph nodes between the hyoid bone and the suprasternal notch. The extent of lymph nodes in level VII were defined as the pretracheal and paratracheal lymph nodes between the suprasternal notch and the innominate vein. 


\section{Surgical procedures}

All patients were treated with general anesthesia and head, back, and neck extension. At two transverse fingers above the superior sternal notch, a collar arc incision (Kocher incision) was made along the direction of the dermatoglyph to cut the skin, subcutaneous tissue, and platysma muscle. The loose connective tissue space of the platysma muscle was separated and the skin flap was freed. The anterior cervical muscle was pulled to both sides to expose the thyroid gland. In the CNs group, CNs (approximately $0.1 \mathrm{~mL}$ per point) were injected with a skin test needle at $1-2$ points around the thyroid carcinoma. The needle was drawn back before injection to confirm that it did not mistakenly enter a blood vessel. The injection site was pressed with gauze immediately after injection to prevent liquid leakage from polluting the operation field. Ten minutes after injection, hemithyroidectomy/total thyroidectomy plus CLND was performed after central lymph nodes were stained black. The procedure in the control group was identical to that in the CNs group, but injection of CNs was omitted. During the operation, care was taken to protect the RLN and inferior PGs. After dissection, the lymph nodes in the central area were sent for pathological examination separately according to level VI and level VII.

\section{Statistical analysis}

The SPSS 25 software package was used for statistical analysis. Normally distributed data were presented as mean \pm standard deviation and the $t$-test was used to compare the difference between two groups. Measurement data that were not normally distributed were presented as the median (M) and interquartile range (Q1, Q3), and the Mann-Whitney $\mathrm{U}$ test was used for comparison between groups. The $\chi^{2}$ test was used to compare the counting data. A $\mathrm{P}$ value $<0.05$ was considered statistically significant.

\section{Results}

\section{Patient characteristics}

Clinical data was retrospectively collated from 191 patients, including 103 patients in the CNs group and 88 patients in the control group. The CNs group included 25 males and 78 females, aged 15 to 82 years, with an average age of 48.57 years. The control group included 21 males and 67 females, aged 18 to 76 years old, with an average age of 45.49 years. The patient clinical characteristics are summarized in Table 1. No significant differences in age, gender, tumor size, number of tumors, capsular invasion, tumor location, and surgical approach were noted between the two groups $(\mathrm{P}>0.05)$.

\section{Lymph node dissection}

Pathological examination post-operation showed that 216 and 134 level VII lymph nodes were dissected in the CNs group and the control group, respectively. In the CNs group, there were 1-5 lymph nodes per case with an average of $2.51 \pm 1.10 \mathrm{lymph}$ nodes per case, and $42 \mathrm{lymph}$ nodes had metastases. In the control group, there were 1-4 lymph nodes per case with an average of $1.97 \pm 0.85$ lymph nodes per case, and 28 lymph nodes had metastases (Table 2). There was no significant difference in the rate of metastasis in level VII lymph nodes between the two groups $(\mathrm{P}=0.741)$. However, the number of lymph node dissections in level VII in the CNs group was significantly greater than that in the control group $(\mathrm{P}=0.001)$. The number of micro lymph nodes $(<5 \mathrm{~mm})$ dissected in level VII in the CNs group was also significantly increased compared with that in the control group $(\mathrm{P}<0.001)$. With the assistance of $\mathrm{CNs}$, the rate of PG detection in level VII was significantly lower compared to the control group (3.88\% versus $12.80 \%)$.

In the CNs group, a total of 216 level VII lymph nodes were dissected, and 151 were stained black (69.91\%). Among the stained lymph nodes, 126 (83.44\%) were stained completely black and $25(16.56 \%)$ were stained partially black. In the CNs group, the PGs identified by clinical experience were not stained black but still maintained the original yellowish-brown or reddish-brown color in sharp contrast with the surrounding black-stained thyroid and lymph nodes (Figure 2). There were 36 lymph nodes that showed metastasis in black-stained lymph nodes, with a metastasis rate of $23.84 \%$. There were only 6 lymph nodes that had metastasis in non-black-stained lymph nodes, with a metastasis rate of $9.23 \%$ (Table 3). The metastatic rate of black-stained lymph nodes was significantly higher than that of non-black-stained lymph nodes $(\mathrm{P}=0.013)$.

\section{Postoperative complications}

Table 4 summarizes the comparison of common postoperative complications between the two groups. In the CNs group, hypoparathyroidism occurred in 8 cases $(7.41 \%)$ as demonstrated by PTH values that were lower than the normal values $(0.34-5.6 \mu \mathrm{IU} / \mathrm{mL})$. Patients in the $\mathrm{CNs}$ 
Table 1 Patient demographics and tumor characteristics

\begin{tabular}{|c|c|c|c|}
\hline Variables & CNs group $(\mathrm{N}=103)$ & Control group ( $\mathrm{N}=88$ ) & $P$ value \\
\hline Gender (male/female) & $25 / 78$ & $21 / 67$ & 0.948 \\
\hline \multicolumn{4}{|l|}{ Tumor size $(\mathrm{cm})$} \\
\hline$\geq 1$ & 19 & 13 & 0.498 \\
\hline \multicolumn{4}{|l|}{ Number of tumors } \\
\hline Single focal & 82 & 72 & 0.701 \\
\hline Multiple focal & 21 & 16 & \\
\hline \multicolumn{4}{|l|}{ Capsular invasion } \\
\hline \multicolumn{4}{|l|}{ Tumor location } \\
\hline Unilateral & 93 & 81 & 0.671 \\
\hline Bilateral & 10 & 7 & \\
\hline \multicolumn{4}{|l|}{ Surgical approach } \\
\hline Hemithyroidectomy plus UCLND & 79 & 71 & 0.504 \\
\hline Total thyroidectomy plus BCLND & 24 & 17 & \\
\hline
\end{tabular}

$\mathrm{P}<0.05$ was considered statistically significant. CNs, carbon nanoparticles; UCND, unilateral central lymph node dissection; BCND, bilateral lymph node dissection.

Table 2 Lymph node involvement and the number of parathyroid glands found in level VII

\begin{tabular}{lcc}
\hline Clinic pathologic data & $\begin{array}{c}\text { CNs group (N=103), N (\%) or } \\
\text { mean } \pm \text { SD }\end{array}$ & $\begin{array}{c}\text { Control group (N=88), N (\%) or } \\
\text { mean } \pm S D\end{array}$ \\
\hline Total number of level VII lymph nodes & 216 & 134 \\
Metastatic level VII lymph nodes & $42(19.4)$ & $28(20.9)$ \\
Non-metastatic level VII lymph nodes & $174(80.6)$ & $106(79.1)$ \\
Dissection number of lymph nodes (mean \pm SD, per patient) & $2.51 \pm 1.10$ & $1.97 \pm 0.85$ \\
Number of lymph nodes $<5$ mm (mean \pm SD, per patient) & $1.58 \pm 0.61$ & $1.07 \pm 0.52$ \\
PGs found in level VII & $4(3.88)$ & $0.001^{*}$ \\
\hline
\end{tabular}

${ }^{*}$, there is statistical difference between two groups $(\mathrm{P}<0.05)$. CNs, carbon nanoparticles; $\mathrm{PG}$, parathyroid gland; $\mathrm{SD}$, standard deviation.

group also experienced symptoms such as numbness around the mouth and extremities. These clinical symptoms were relieved after intravenous injection of calcium gluconate. Postoperative hypoparathyroidism occurred in 17 cases $(19.32 \%)$ in the control group, which was significantly higher than the number of cases in the CNs group $(\mathrm{P}=0.018)$. Again, treatment with calcium gluconate alleviated the symptoms.
During the follow-up within 6 months after the operation, the PTH levels of all patients returned to normal, and no permanent hypoparathyroidism was observed.

There was 1 case of RLN injury in the CNs group $(0.97 \%)$ and 3 cases in the control group (3.41\%). The difference was not statistically significant $(\mathrm{P}=0.505)$. Patients with RLN injury exhibited hoarseness and were 

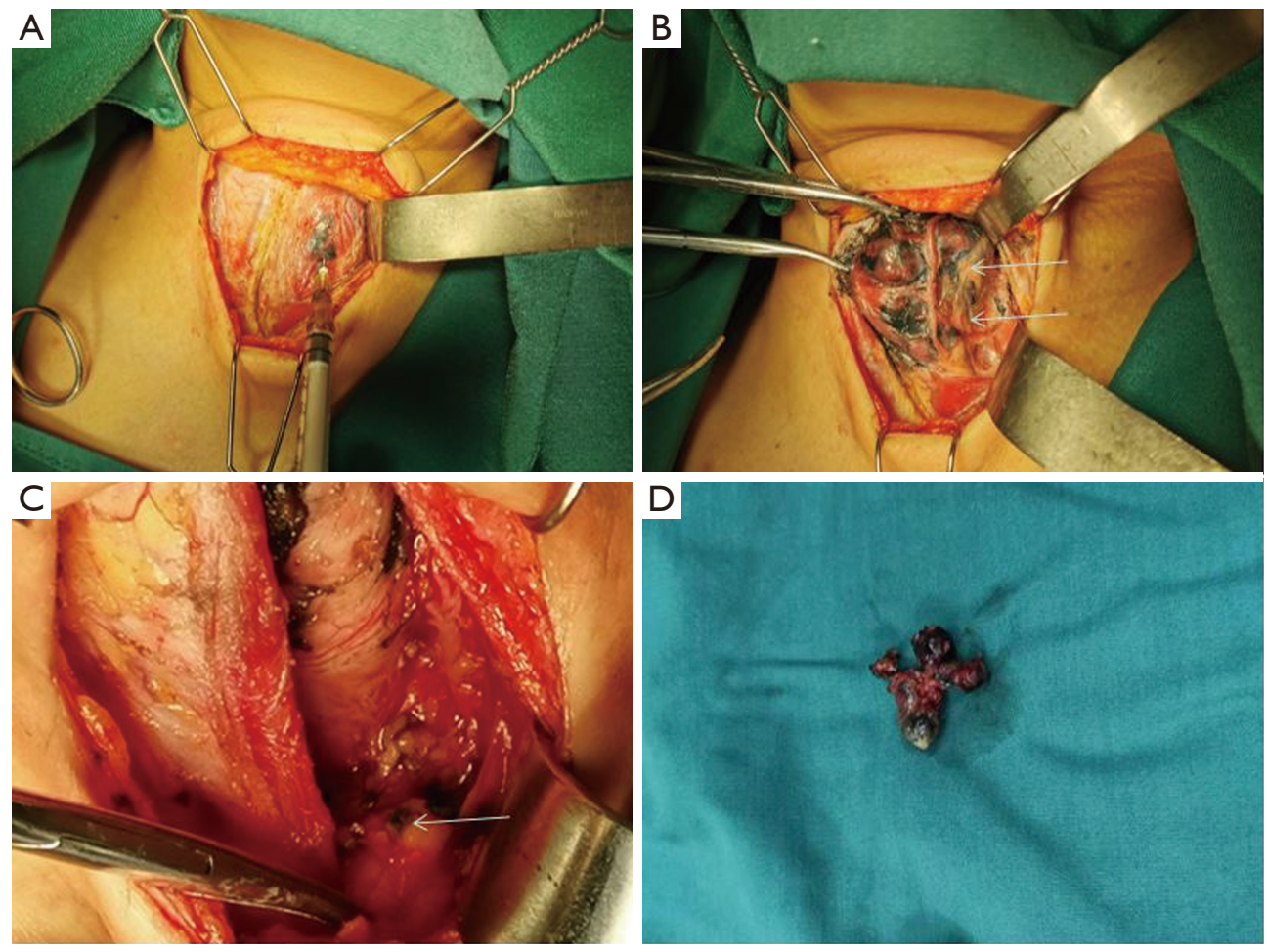

Figure 2 The procedure of injecting CNs into thyroid tissue. The thyroid tissue, lymph nodes are stained black and the PGs remain the original color. (A) The injection of carbon nanoparticles into thyroid tissue; (B) the arrows point to the parathyroid glands; (C) the arrow points to the level VII lymph node; (D) the central region lymph nodes.

Table 3 Level VII lymph node involvement in the carbon nanoparticle group

\begin{tabular}{|c|c|c|c|c|}
\hline Variables & Black-stained, N (\%) & Non-black-stained, N (\%) & Total & $P$ value \\
\hline Metastatic lymph nodes in level VII & $36(85.7)$ & $6(14.3)$ & 42 & - \\
\hline
\end{tabular}

*, there is statistical difference between two groups $(P<0.05)$.

treated with mecobalamin. All patients were examined by electronic laryngoscopy 6 months after the operation. The movement of vocal cords returned to normal, and no permanent vocal cord paralysis was noted. There were no significant differences in the incidence of other common complications, such as postoperative bleeding and superior laryngeal nerve injury, between the two groups $(\mathrm{P}>0.05)$. None of the patients experienced dyspnea, bilateral RLN injury, chylous fistula, nor pneumonia. The patients were followed up for 6 months, and no recurrence, metastasis, nor death was observed.

\section{Other inpatient-related indicators}

Intraoperative blood loss, operation duration, postoperative drainage duration, and hospitalization days were comparable between the two groups with no statistically significant differences $(\mathrm{P}>0.05)$. However, the whole hospitalization cost of the $\mathrm{CNs}$ group was significantly higher compared to the control group $(\mathrm{P}<0.001$; Table 5$)$.

\section{Discussion}

In recent years, various types of lymph node tracers have 
Table 4 Postoperative complications

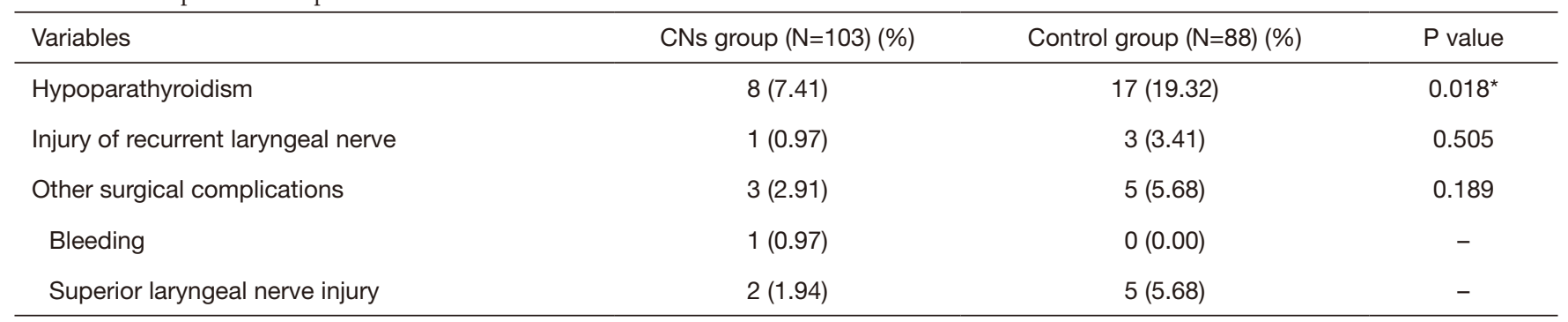

*, there is statistical difference between two groups $(P<0.05)$. CNs, carbon nanoparticles.

Table 5 Other inpatient-related indicators

\begin{tabular}{|c|c|c|c|}
\hline Variables & CNs group $(\mathrm{N}=103)$ & Control group $(\mathrm{N}=88)$ & $P$ value \\
\hline Operation duration ( $\bar{x} \pm \mathrm{s}, \mathrm{min})$ & $85.45 \pm 32.91$ & $89.13 \pm 36.62$ & 0.891 \\
\hline Postoperative drainage duration $(\bar{x} \pm \mathrm{s}, \mathrm{d})$ & $2.4 \pm 1.7$ & $2.6 \pm 1.3$ & 0.929 \\
\hline Hospitalization days $(\bar{x} \pm \mathrm{s}, \mathrm{d})$ & $4.1 \pm 2.9$ & $5.5 \pm 4.6$ & 0.544 \\
\hline
\end{tabular}

${ }^{*}$, there is statistical difference between two groups $(P<0.05)$. CNs, carbon nanoparticles.

been developed to recognize the characteristics of level VII lymph nodes, including indocyanine green, methylene blue, and India ink (15). CNs are an advanced tracer that can be used in PTC surgeries. These lymphatic tracers have a particle diameter of $150 \mathrm{~nm}$. CNs tend to congregate in the lymphatic system and do not enter blood vessels. Due to lymphoid aggregation, CNs have been useful for the detection of level VII lymph nodes and facilitate thorough clearing of lymph nodes in the central area.

In this current study, the number of level VII lymph nodes detected in the CNs group was significantly higher compared to the control group, but the rate of lymph node metastases was not significantly different between the two groups. These results suggested that $\mathrm{CNs}$ can be used to effectively increase the detection rate of lymph nodes during surgery and contribute to a more thorough dissection of cervical lymph nodes, especially lymph nodes that cannot be detected by ultrasound. The increased rate of lymph node detection could be explained by several reasons. First, some micro-lymph nodes with a diameter less than $5 \mathrm{~mm}$ can be found in level VII (16). However, due to their small volume, these lymph nodes are often missed by surgeons. CNs have a good tracer effect and can help identify and remove these micro-lymph nodes. Second, CNs can also indicate the drainage direction of lymphatic vessels in the tissue, which can assist surgeons expand the scope of the surgical dissection during the operation. Third, the tracing effect of CNs make it easier and more accurate for pathologists to obtain lymph node specimens during the operation and reduce the influence of human factors on the number of lymph nodes. Therefore, in patients with PTC, the use of CNs can reduce the omission of lymph nodes in the central area, help to reduce the local recurrence rate after operation, provide more accurate clinical staging, and reduce the amount of postoperative radioactive iodine ablation.

This investigation demonstrated that the lymph node metastasis rate of black-stained lymph nodes was significantly higher than that of non-black-stained lymph nodes, which suggested that $\mathrm{CNs}$ black staining had higher detection efficiency. It is worth mentioning that the lymph node metastasis rate of black-stained lymph nodes during CLND has been reported to be as high as $83-100 \%(17-21)$, which differs from the results of this current study. One reason for this difference may be that all discernible level VII lymph nodes were cleared during our operations, whereas other studies exclusively focused on suspicious lymph nodes observed by ultrasound prior to the operation. Another reason may be that lymph node dissections mainly occurred in level VI in other investigations, and the 
metastasis rate of level VI lymph nodes was approximately $20-90 \%(6,7,22)$, which is higher than that in level VII lymph nodes (16-38\%) (8).

Hypoparathyroidism is a common complication of thyroid cancer surgery with a reported incidence of 6.9$46.5 \%(23,24)$. In the presence of PG anatomical variation and when PGs are mixed with the surrounding lymphoid node and adipose tissue, the PGs may be mistaken for lymph nodes and be resected during CLND. Conversely, it is also possible that lymph nodes are mistaken as PGs, thus resulting in incomplete dissection. In addition, after CLND, the disturbance of the blood supply to PGs is the primary cause of postoperative hypoparathyroidism. Unfortunately, chemical and physical methods or biological dyes that can label PGs during operations are currently not available. With the application of $\mathrm{CNs}$, the thyroid glands and its surrounding lymph nodes are stained black, but no black staining is found in PGs or the RLN, thus achieving a negative parathyroid staining effect. This is helpful in identifying and protecting PGs during operations (25-27). In this study, the transient hypocalcemia rate observed in the CNs group was significantly reduced compared to that in the control group. After the same calcium supplement treatment, the symptoms alleviated and the blood calcium levels returned to normal. During the 6-month follow-up period, no permanent hypoparathyroidism was observed.

The disadvantage of using CNs is that the whole hospitalization cost is significantly elevated, and this may present an affordability issue for some patients. Unfortunately, the follow-up time in this investigation was too short to effectively examine the ability of CNs to reduce the recurrence rate in level VII in patients with PTC. Future studies with a longer follow-up period are warranted.

\section{Conclusions}

The use of CNs for lymph node tracing can significantly increase the dissected number of level VII lymph nodes and help to protect the PGs, thereby achieving a more radical treatment effect. Moreover, the use of $\mathrm{CNs}$ does not increase the rate of injury to the RLN nor the rate of other complications which confirmed the safety for patients. CNs are also advantageous as they do not require any special preparation, and provide a effective approach for level VII dissection in patients with PTC.

\section{Acknowledgments}

Funding: This study was supported by the Young and Middle-aged Teachers Education Research Project of Fujian Province (JAT200503).

\section{Footnote}

Reporting Checklist: The authors have completed the STROBE reporting checklist. Available at https://dx.doi. org/10.21037/gs-21-281

Data Sharing Statement: Available at https://dx.doi. org/10.21037/gs-21-281

Conflicts of Interest: All authors have completed the ICMJE uniform disclosure form (available at https://dx.doi. org/10.21037/gs-21-281). The authors have no conflicts of interest to declare.

Ethical Statement: The authors are accountable for all aspects of the work in ensuring that questions related to the accuracy or integrity of any part of the work are appropriately investigated and resolved. All procedures performed in this study involving human participants were in accordance with the Declaration of Helsinki (as revised in 2013). This research was approved by the medical ethics committee of the Affiliated Hospital of Putian University. All patients were informed of the methods and risks of the operation and signed informed consents.

Open Access Statement: This is an Open Access article distributed in accordance with the Creative Commons Attribution-NonCommercial-NoDerivs 4.0 International License (CC BY-NC-ND 4.0), which permits the noncommercial replication and distribution of the article with the strict proviso that no changes or edits are made and the original work is properly cited (including links to both the formal publication through the relevant DOI and the license). See: https://creativecommons.org/licenses/by-nc-nd/4.0/.

\section{References}

1. Bray F, Ferlay J, Soerjomataram I, et al. Global cancer statistics 2018: GLOBOCAN estimates of incidence and mortality worldwide for 36 cancers in 185 countries. CA 
Cancer J Clin 2018;68:394-424.

2. Haugen BR, Alexander EK, Bible KC, et al. 2015 American Thyroid Association Management Guidelines for adult patients with thyroid nodules and differentiated thyroid cancer: the American Thyroid Association Guidelines Task Force on thyroid nodules and differentiated thyroid cancer. Thyroid 2016;26:1-133.

3. Mazzaferri EL, Jhiang SM. Long-term impact of initial surgical and medical therapy on papillary and follicular thyroid cancer. Am J Med 1994;97:418-28.

4. Akın Ş, Yazgan Aksoy D, Akın S, et al. Prediction of central lymph node metastasis in patientswith thyroid papillary microcarcinoma. Turk J Med Sci 2017;47:1723-7.

5. Lee JH, Shin HJ, Yoon JH, et al. Predicting lymph node metastasis in patients with papillary thyroid carcinoma by vascular index on power Doppler ultrasound. Head Neck 2017;39:334-40.

6. Ito Y, Miyauchi A. Lateral and mediastinal lymph node dissection in differentiated thyroid carcinoma: indications, benefits, and risks. World J Surg 2007;31:905-15.

7. Davidson HC, Park BJ, Johnson JT. Papillary thyroid cancer: controversies in the management of neck metastasis. Laryngoscope 2008;118:2161-5.

8. Wang LY, Versnick MA, Gill AJ, et al. Level VII is an important component of central neck dissection for papillary thyroid cancer. Ann Surg Oncol 2013;20:2261-5.

9. Tavares MR, Michaluart P Jr, Montenegro F, et al. Skip metastases in medullary thyroid carcinoma: a single-center experience. Surg Today 2008;38:499-504.

10. Tavares MR, Cruz JA, Waisberg DR, et al. Lymph node distribution in the central compartment of the neck: an anatomic study. Head Neck 2014;36:1425-30.

11. Hughes DT, Doherty GM. Central neck dissection for papillary thyroid cancer. Cancer Control 2011;18:83-8.

12. Shaha AR, Migliacci JC, Nixon IJ, et al. Stage migration with the new American Joint Committee on Cancer (AJCC) staging system (8th edition) for differentiated thyroid cancer. Surgery 2019;165:6-11.

13. Chaojie Z, Shanshan L, Zhigong Z, et al. Evaluation of the clinical value of carbon nanoparticles as lymph node tracer in differentiated thyroid carcinoma requiring reoperation. Int J Clin Oncol 2016;21:68-74.

14. Gao B, Tian W, Jiang Y, et al. Application of carbon nanoparticles for parathyroid protection in reoperation of thyroid diseases. Int J Clin Exp Med
2015;8:22254-61.

15. Ryan WR, Orloff LA. Intraoperative tumor localization with surgeon-performed ultrasound-guided needle dye injection. Laryngoscope 2011;121:1651-5.

16. Sessa L, Lombardi CP, De Crea C, Tempera SE, Bellantone R, Raffaelli M. Risk factors for central neck lymph node metastases in micro- versus macro-clinically node negative papillary thyroid carcinoma. World J Surg 2018;42:623-9.

17. Chami L, Hartl D, Leboulleux S, et al. Preoperative localization of neck recurrences from thyroid cancer: charcoal tattooing under ultrasound guidance. Thyroid 2015;25:341-6.

18. Soprani F, Bondi F, Puccetti M, et al. Charcoal tattoo localization for differentiated thyroid cancer recurrence in the central compartment of the neck. Acta Otorhinolaryngol Ital 2012;32:87-92.

19. Kang TW, Shin JH, Han BK, et al. Preoperative ultrasound-guided tattooing localization of recurrences after thyroidectomy: safety and effectiveness. Ann Surg Oncol 2009;16:1655-9.

20. Hartl DM, Chami L, Al Ghuzlan A, et al. Charcoal suspension tattoo localization for differentiated thyroid cancer recurrence. Ann Surg Oncol 2009;16:2602-8.

21. Kwon H, Tae SY, Kim SJ, et al. Role of charcoal tattooing in localization of recurred papillary thyroid carcinoma: initial experiences. Ann Surg Treat Res 2015;88:140-4.

22. Cho SY, Lee TH, Ku YH, et al. Central lymph node metastasis in papillary thyroid microcarcinoma can be stratified according to the number, the size of metastatic foci, and the presence of desmoplasia. Surgery 2015;157:111-8.

23. Cavicchi O, Piccin O, Caliceti U, et al. Accuracy of PTH assay and corrected calcium in early prediction of hypoparathyroidism after thyroid surgery. Otolaryngol Head Neck Surg 2008;138:594-600.

24. Bellantone R, Lombardi CP, Raffaelli M, et al. Is routine supplementation therapy (calcium and vitamin D) useful after total thyroidectomy? Surgery 2002;132:1109-12; discussion 1112-3.

25. Shi C, Tian B, Li S, et al. Enhanced identification and functional protective role of carbon nanoparticles on parathyroid in thyroid cancer surgery: a retrospective Chinese population study. Medicine (Baltimore) 2016;95:e5148.

26. Su AP, Wei T, Liu F, et al. Use of carbon nanoparticles 
to improve the dissection of lymph nodes and the identification of parathyroid glands during thyroidectomy for papillary thyroid cancer. Int J Clin Exp Med 2016;9:19529-36.

27. Shang J, Gu J, Wang W, et al. Potential role for carbon nanoparticles identification and preservation in situ of parathyroid glands during total thyroidectomy and central compartment node dissection. J Clin Oncol 2015;33:e17048.

(English Language Editor: J. Teoh)

Cite this article as: Chen Y, Zhang G, Lin Y, Zhang G, Gao J. The advantages of carbon nanoparticles in level VII lymph node dissection in patients with papillary thyroid cancer. Gland Surg 2021;10(6):2028-2036. doi: 10.21037/gs-21-281 